\title{
Lymph node evaluation in endometrial cancer: how did it change over the last two decades?
}

\author{
Ciro Pinelli ${ }^{1}$, Valeria Artuso ${ }^{1}$, Giorgio Bogani ${ }^{2}$, Antonio Simone Laganà ${ }^{1}$, Fabio Ghezzi ${ }^{1}$, Jvan Casarin ${ }^{1}$ \\ ${ }^{1}$ Obstetrics and Gynecology Department, the University of Insubria, Varese, Italy; ${ }^{2}$ Fondazione IRCCS Istituto Nazionale dei Tumori, Milano, Italy \\ Contributions: (I) Conception and design: C Pinelli, F Ghezzi, J Casarin; (II) Administrative support: F Ghezzi; (III) Provision of study materials or \\ patients: C Pinelli, V Artuso, J Casarin; (IV) Collection and assembly of data: C Pinelli, V Artuso; (V) Data analysis and interpretation: C Pinelli, G \\ Bogani, J Casarin; (VI) Manuscript writing: All authors; (VII) Final approval of manuscript: All authors. \\ Correspondence to: Ciro Pinelli, MD. Obstetrics and Gynecology Department, the University of Insubria, Varese, Italy. Women's and Children Del \\ Ponte Hospital, 21100 Varese, Italy. Email: ciropinelli88@gmail.com.
}

\begin{abstract}
Endometrial cancer (EC) is the most common gynecological malignancy in developed countries, and surgery represents the pivotal part of treatment. Hysterectomy and salpingo-oophorectomy allow removing the primary tumor and defining patients at higher risk, who might benefit from adjuvant therapies. Minimally invasive surgery is associated with superior postoperative outcomes and represents a safe and effective approach for surgical staging of EC. The lymph node status evaluation in EC is still a matter of debate. Over the last twenty years much has changed, moving from a full systematic pelvic and paraaortic lymphadenectomy for staging purpose to the removal of the pelvic (with or without paraaortic) lymph nodes only in selected EC classes of risk. Two randomized trials failed to demonstrate survival benefits of lymphadenectomy in case of apparent early stage EC; however, its prognostic role has never been questioned. At present, with the aim of reducing the surgical-related morbidity, sentinel node mapping is emerging as a safe and valid alternative to lymphadenectomy for EC staging, demonstrating high accuracy and an increased detection of lymph nodes metastasis. Here, we performed a review of the most significant studies, which supported the changes in the lymph node status evaluation for EC over the last two decades.
\end{abstract}

Keywords: Endometrial cancer (EC); sentinel node mapping; lymphadenectomy

Submitted May 23, 2020. Accepted for publication Jul 31, 2020.

doi: $10.21037 /$ tcr-20-2165

View this article at: http://dx.doi.org/10.21037/tcr-20-2165

\section{Introduction}

Endometrial cancer (EC) is the most common gynecological malignancy in developed countries, with more than 65,000 new cases estimated for the year 2020 in the United States (1). The incidence has been rising in the last decades worldwide, due to changing lifestyle and socioeconomic factors, including obesity, one of the most important risk factor (2).

$\mathrm{EC}$ is generally diagnosed in post-menopausal age, nearly half of the patients are over 65 years, which often makes it a challenging treatment, requiring a multi-disciplinary approach (3).

However $10-15 \%$ of EC occur in women younger than
50 years old, often with family or personal history of cancer, indicative of Lynch syndrome (4).

In more than three-quarters of patients with EC the disease is confined to the uterine corpus at the time of primary diagnosis, and the pivotal part of the treatment is represented by the surgical removal of the uterus and the adnexa (5). Traditionally, open abdominal surgery was considered the standard approach for women with apparent early stage disease. More recently, the implementation of the minimally invasive surgery, first laparoscopy (6), and subsequently, robotic-assisted surgery (7) brought several advantages for the treatment of apparent early stage EC, such as shorter hospitalization, less complications, and reduced costs. 
Regarding the oncological outcomes, two randomized controlled trials, LACE and LAP2 trials, comparing laparoscopy and laparotomy in early-stage EC, showed no significant differences in the risk of disease recurrence and death $(8,9)$. For these reasons minimally invasive surgery, to date, should be considered the preferable approach in the treatment of EC patients. Vaginal approach might be considered in selected patients, usually medically unfit for either laparoscopic or open surgery (10).

Preoperative workup of patients with EC should include pathological information, to define histotype and grading of tumor, and imaging assessment to exclude extrauterine disease and to describe myometrial or cervical stroma infiltration. Although there has been a constant improvement of the imaging techniques and dedicated pathologists, the preoperative accuracy of EC staging still remain an unsolved issue. Previous published studies have underlined a discrepancy rate higher than $30 \%$ between preoperative risk assessment, based on biopsy (pipelle device or operative hysteroscopy) and MRI, and the actual risk of final pathological finding (11). These discrepancies might open two scenarios: in one case an overestimation could occur with unnecessary lymphadenectomy, on the other hand patients could result potentially under staged, needing for further therapies.

Recently, genetic serum biomarkers have been investigated for early detection of EC, but these are still experimental. (12)

Preoperative CT scan has limited benefit in low-risk EC; conversely it is useful in G3 endometrioid histotype and type $2 \mathrm{EC}$ to detect gross intrabdominal disease and enlarged nodes, specifically in the paraaortic area (13). MRI is currently recommended for pre-operative evaluation in some guidelines and studies (14), however expert ultrasound assessment is supported as a routine procedure in the preoperative assessment of EC in order to infer the myometrial invasion and cervical involvement or synchronous ovarian cancer (15). PET-CT scan has been described as the best imaging method to evaluate lymph node and distant metastases in high-risk or advanced stage EC, however, its sensitivity is not sufficient to justify its routine use for preoperative staging in case of apparent early stage disease $(16,17)$.

Moreover, glandular cells detected at preoperative cervical-smear could be able to predict risk of local recurrence in EC but further studies are needed to confirm these results (18).

A crucial step of the surgical staging of EC is the evaluation of the lymph node status. Approximately $10 \%$ of women presenting with apparent early stage EC, as established by preoperative workup, will be found to have lymph node metastases, the most common site of extrauterine spread (19). Different studies have investigated the factors associated with lymph node metastasis: the nonendometrioid histology, worsening tumor grade, increasing depth of myometrial invasion and lymphovascular space invasion have been shown the main predictors of positive nodes $(20,21)$.

Below we reported the changes in the lymph node status evaluation which happened over the last two decades and the strategies developed to decrease the morbidity for this specific step of the surgical staging.

\section{From full to selective lymph node evaluation}

Over the last decades, one of the main concerns in the management of EC has been lymph node evaluation: particularly referring to the need and the extent of lymphadenectomy and its therapeutic role.

Traditionally, on the basis of FIGO recommendations, systematic pelvic and paraaortic lymphadenectomy was routinely performed for EC staging (5).

Later in time, Mariani et al. proposed to omit lymphadenectomy in patients with endometrioid EC with myometrial invasion less than $50 \%$, grade $1-2$ tumors, tumor diameter less than $2 \mathrm{~cm}$, and no evidence of extrauterine disease, observing a 5 -year cancer-specific survival of $97 \%$ (22). On the other hand, all the other patients were considered at risk of lymph node metastasis and were candidated for systematic pelvic and paraaortic lymphadenectomy up to the renal vessels, because of the high frequency (about $50 \%$ ) of simultaneous involvement in the para-aortic area of the patients who had pelvic lymphatic metastases and to identify isolated paraaortic positive nodes (generally $\leq 5 \%$ ) (23).

Evidence in favor of the therapeutic role of lymphadenectomy were based on retrospective studies such as the SEPAL study, which included more than 600 patients, showing survival benefit of systematic pelvic and para-aortic lymphadenectomy over pelvic lymphadenectomy alone in intermediate- and high-risk EC, while no difference was proven in low-risk EC (24). However, evidence coming from two randomized trials (ASTEC trial and an Italian collaborative trial by Benedetti Panici et al.) and a metanalysis failed to demonstrate survival benefits of lymphadenectomy in patients with apparent early-stage EC, 
although several criticisms in the study design of both trials have been made (25-27).

According to these trials, the retroperitoneal staging with the removal of pelvic and paraaortic lymph nodes maintained only a prognostic relevance to tailor the postoperative adjuvant therapy in patients with positive nodes, in particular chemotherapy.

At present, the European Society of Medical Oncology (ESMO)/European Society of Gynaecological Oncology (ESGO)/European Society for Radiotherapy \& Oncology (ESTRO) guidelines support the performance of lymphadenectomy as an integral part of comprehensive staging in patients with apparent early-stage EC (except for low-risk EC, grade 1 or 2 and myometrial invasion $<50 \%)(10)$.

Mirroring the shift from laparotomy to laparoscopy, in order to reduce the morbidity of surgical staging, in the last decades sentinel lymph node (SLN) mapping has emerged as a valid alternative to lymphadenectomy in EC among gynecologic oncologists (28). Complete pelvic and paraaortic lymphadenectomy may be correlated with severe surgical morbidities such as lymph cyst, lymphedema, massive bleeding, and urologic, nerves or vascular injury. SLN detection is improving the management of women with early EC avoiding them unnecessary and potentially injurious systematic lymphadenectomies, all the while obtaining information about lymph node status.

\section{Sentinel node mapping}

Most of the early retrospective series of SLN in EC were published during first decade of this millennium, while in 2011 Ballester et al. published the results of SENTIENDO, the first prospective multicenter study (29). EC patients underwent SLN biopsy followed by complete pelvic lymphadenectomy, to estimate the accuracy of this technique. The results were very encouraging: overall detection rate was $82 \%$ and sensitivity was $100 \%$ for hemipelvis, without any false negative. A year later this experience, Abu-Rustum and his group introduced a SLN mapping algorithm (MSKCC algorithm) which consisted in a lymph nodes evaluation including excision of all mapped SLNs and removal of all suspicious nodes regardless of mapping. If there was no mapping on a hemipelvis, a sidespecific pelvic lymphadenectomy was performed. This algorithm, retrospectively applied, has shown sensitivity and negative predictive value near to $100 \%$ and a low falsenegative rate of $1.9 \%$ (30).
Several techniques and tracers were analyzed for the SLN mapping in EC. Technetium sulphur colloid, alone or in combination with blue dye, was historically adopted and radioactive lymph nodes were identified using a gamma-probe (31). Indocyanine Green (ICG), a contrast agent which turns fluorescent when exposed to near-infrared light, is a more recent option for lymphatic mapping (32).

ICG is related with the highest SLN detection rate: results from the recent FILM trial showed superior capabilities compared to blue dye (33). The better performance and the simplicity of ICG detection make it preferable to other modalities.

Two main sites of tracer injection for SLN mapping in EC have been described: intracervical and endometrial injection via hysteroscopy. Cervical injection is the most used method: it is easily accessible and can be injected accurately with few additional equipment and it is associated with high pelvic detection rate and accuracy (34). However, mapping using cervical dye injection was mainly criticized because it does not adequately map the paraaortic area and may potentially miss cases of isolated paraaortic disease (35).

In 2017, the accuracy of SLN in EC was confirmed by the results of FIRES trials, an American multicenter prospective cohort study, which investigated sensitivity and negative predictive value of SLN mapping, using a standardized technique through cervical injection of ICG tracer, compared with systematic lymphadenectomy in detecting nodes metastasis (36). Nodal metastases were identified in the SLNs of 35 of 36 patients, giving a sensitivity of $97.2 \%$ and a negative predictive value of $99.6 \%$, confirming the high diagnostic accuracy in detecting EC nodal metastases, possibly replacing lymphadenectomy in the staging of EC.

The main concern about SLN was its safety in high grade EC, associated with higher risk of nodes metastasis. The biggest prospective study about high risk patients (grade 3 endometrioid and type 2 EC) treated with SLN mapping followed by full pelvic and para-aortic lymphadenectomy supported the use of SLN even in this subgroup of patients, showing high sensitivity and negative predictive value (37).

The latest NCCN Guidelines (version 1.2020) approved SLN algorithm for surgical staging of EC, regardless of risk class; the sentence "is under evaluation", previously included regarding the role of SLN mapping, was omitted in this version. 


\section{Ultrastaging}

SLNs removed are subjected to pathological ultrastaging, which consists in a more sensitive and precise identification of nodes disease, by immunohistochemistry (IHC), than conventional routine hematoxylin and eosin (H\&E) technique. This technique succeeded in identifying low volume metastasis: micrometastasis and isolated tumor cells (microscopic clusters and single neoplastic cells measuring $>0.2 \mathrm{~mm}$ to $\leq 2 \mathrm{~mm}$ and $\leq 0.2 \mathrm{~mm}$, respectively).

Low volume metastasis are far from rare: FIRES and the FILM trials reported a percentage in SLN of 54\% and $62 \%$, respectively, and generally account for one third of positive nodes in EC patients (38). Micrometastasis and isolated tumor cells are more often diagnosed in the lowrisk than in the high-risk group, in which adjuvant therapy is generally omitted.

The Mayo Clinic (Rochester, MN) and the MSKCC (NY), compared outcomes of patients with low risk EC treated with two different strategies according to their institution protocol: SLN mapping algorithm at MSKCC and full pelvic and paraaortic lymphadenectomy in cases considered at risk for nodal metastasis (grading 3 and/ or tumor diameter $>2 \mathrm{~cm}$ ) at Mayo Clinic. Pelvic nodes metastases (including low volume metastases) were detected in $5.1 \%$ and $2.6 \%$ of patients respectively $(\mathrm{P}=0.03)$. Therefore, SLN mapping was found to improve the detection of nodal disease in comparison to conventional lymphadenectomy, probably due to ultrastaging, which allows the identification of low volume disease not evident with conventional histological examination. Disease free survival and overall survival at 3 years were similar between the two groups (39).

Moreover, a recent study demonstrates that, in patients with isolated paraaortic dissemination, ultrastaging of pelvic lymph nodes allowed the identification of low-volume disease, reducing the prevalence of true isolated paraaortic disease (40).

Actually, prognostic value of low volume nodes metastasis is not clear, a recent metanalysis showed higher risk of recurrence in patients with low volume metastasis, regardless of adjuvant therapy administration (41).

The use of adjuvant treatments is a topic of debate and should consider uterine factors for patients with isolated tumor cells, while could be administered for patients with micrometastases (38).

In fact, data coming from retrospective studies showed better survival outcomes in patients with isolated tumor cells (most of whom were treated with adjuvant therapy) compared to those with macrometastases, recurrences were similar to patients without metastatic disease (42).

\section{Frozen section}

Intraoperative pathological examination could improve EC patient risk classification and, thus, plays an important role in the evaluation of surgical decision, mainly when preoperative assessment is not conclusive. The frozen endometrial tissue, in fact, was used for a long time to identify patients with low risk EC according to "Mayo criteria", avoiding them systematic lymphadenectomy (43).

However, the introduction of SLN for EC staging regardless tumor risk factors reduced the need for intraoperative evaluation, which requires higher operative time and costs.

NCCN guidelines suggest evaluation of the SLNs removed with ultrastaging, therefore frozen section analysis of the removed lymph nodes is not routinely performed.

However, the use of frozen section could be adopted in order to make sure to identify the presence of lymph nodes in the SLN to properly assess lymph node status, since a recent study reported a percentage of $8 \%$ of SLN removed as an 'empty node' at final pathology, consisting of fibroadipose tissue in the absence of lymphatic tissue (44).

\section{Oncological outcomes of SLN}

Some studies suggested that SLN mapping provides similar oncologic outcomes in comparison to systematic lymphadenectomy, however, the evidence is still limited to retrospective investigations.

A meta-analysis of about 3,500 patients showed higher positive pelvic nodal detection rates, similar paraaortic nodal detection rates, and no difference in overall recurrence or nodal recurrence rates in SLN compared with systematic lymphadenectomy (45).

These data suggested that SLN biopsy may actually increase the detection of metastatic disease (particularly in low-risk group), resulting in optimal adjuvant therapy prescription, without compromising survival outcomes.

Moreover, in a recent multi-institutional Italian retrospective study which evaluated long-term outcomes of EC patients who underwent lymphadenectomy, SLN mapping followed by lymphadenectomy and SLN mapping alone, the survival comparison of the three techniques did not show differences of disease-free and overall survival, 
also after stratification by low, intermediate and high risk patients (46).

\section{Future perspectives}

At present, the risk stratification systems used to guide the need for adjuvant treatment of EC are mainly based on the post-surgical pathological information. Recently, with the use of microarray and sequencing technologies, the Cancer Genome Atlas (TCGA) showed four molecular subgroups in EC, which were validated and simplified as follows: POLE ultramutated, mismatch repair-deficient, p53 mutant and those EC lacking any of these alterations (47).

The ongoing PORTEC4a trial is the first clinical setting which investigates the incorporation of molecular parameters into the current clinicopathological classification in adjuvant treatment decisions (48). This shift towards a molecular driven EC classification is an important step to the future precision medicine.

\section{Conclusions}

EC surgical staging has been changing over the last twenty years. Minimally invasive techniques became the principal surgical approach in case of apparent early stage disease, since associated with less morbidity and noninferior survivals in comparison with open surgery. On the other hand, the role of the retroperitoneal staging still represents a grey area, and remains matter of debate among gynecologic oncologists, worldwide. However, the results of the recent studies fully supported the adoption of the SLN technique, since reducing the surgical-related morbidity, without impacting on survival, even in case of high-risk cancers. Clinicians should follow the indication of the most recent national and international guidelines, with the aim of offering adequate and homogeneous treatment to all EC patients.

\section{Acknowledgments}

Funding: None.

\section{Footnote}

Provenance and Peer Review: This article was commissioned by the Guest Editor (Antonio Simone Laganà) for the series "Endometrial Cancer" published in Translational Cancer Research. The article was sent for external peer review organized by the Guest Editor and the editorial office.

Conflicts of Interest: All authors have completed the ICMJE uniform disclosure form (available at http://dx. doi. org/10. 21037/tcr-20-2165). The series "Endometrial Cancer" was commissioned by the editorial office without any funding or sponsorship. ASL served as the unpaid Guest Editor of the series, and serves as an unpaid editorial board member of Translational Cancer Research from Dec 2019 to Nov 2021. The authors have no other conflicts of interest to declare.

Ethical statement: the authors are accountable for all aspects of the work in ensuring that questions related to the accuracy or integrity of any part of the work are appropriately investigated and resolved.

Open Access Statement: This is an Open Access article distributed in accordance with the Creative Commons Attribution-NonCommercial-NoDerivs 4.0 International License (CC BY-NC-ND 4.0), which permits the noncommercial replication and distribution of the article with the strict proviso that no changes or edits are made and the original work is properly cited (including links to both the formal publication through the relevant DOI and the license). See: https://creativecommons.org/licenses/by-nc-nd/4.0/.

\section{References}

1. Siegel RL, Miller KD, Jemal A. Cancer statistics, 2020. CA Cancer J Clin 2020;70:7-30.

2. Constantine GD, Kessler G, Graham S, et al. Increased Incidence of Endometrial Cancer Following the Women's Health Initiative: An Assessment of Risk Factors. J Womens Health (Larchmt) 2019;28:237-43.

3. Vitale SG, Capriglione S, Zito G, et al. Management of endometrial, ovarian and cervical cancer in the elderly: current approach to a challenging condition. Arch Gynecol Obstet 2019;299:299-315.

4. Gruber SB, Thompson WD. A population-based study of endometrial cancer and familial risk in younger women. Cancer and Steroid Hormone Study Group. Cancer Epidemiol Biomarkers Prev 1996;5:411-7.

5. Creasman WT, Odicino F, Maisonneuve P, et al. Carcinoma of the corpus uteri. FIGO 26th Annual Report on the Results of Treatment in Gynecological Cancer. Int J Gynaecol Obstet 2006;95 Suppl 1:S105-43.

6. Childers JM, Brzechffa PR, Hatch KD, et al. 
Laparoscopically assisted surgical staging (LASS) of endometrial cancer. Gynecol Oncol 1993;51:33-8.

7. Boggess JF. Robotic surgery in gynecologic oncology: evolution of a new surgical paradigm. J Robot Surg 2007;1:31-7.

8. Janda M, Gebski V, Davies LC, et al. Effect of Total Laparoscopic Hysterectomy vs Total Abdominal Hysterectomy on Disease-Free Survival Among Women With Stage I Endometrial Cancer: A Randomized Clinical Trial. JAMA 2017;317:1224-33.

9. Walker JL, Piedmonte MR, Spirtos NM, et al. Recurrence and survival after random assignment to laparoscopy versus laparotomy for comprehensive surgical staging of uterine cancer: Gynecologic Oncology Group LAP2 Study. J Clin Oncol 2012;30:695-700.

10. Colombo N, Creutzberg C, Amant F, et al. ESMOESGO-ESTRO Consensus Conference on Endometrial Cancer: diagnosis, treatment and follow-up. Ann Oncol 2016;27:16-41.

11. Body N, Lavoué V, De Kerdaniel O, et al. Are preoperative histology and MRI useful for classification of endometrial cancer risk? BMC Cancer 2016;16:498.

12. Benati M, Montagnana M, Danese E, et al. Aberrant Telomere Length in Circulating Cell-Free DNA as Possible Blood Biomarker with High Diagnostic Performance in Endometrial Cancer. Pathol Oncol Res 2020;26:2281-9.

13. Bogani G, Gostout BS, Dowdy SC, et al. Clinical Utility of Preoperative Computed Tomography in Patients With Endometrial Cancer. Int J Gynecol Cancer 2017;27:1685-93.

14. Cignini P, Vitale SG, Laganà AS, et al. Preoperative workup for definition of lymph node risk involvement in early stage endometrial cancer: 5-year follow-up. Updates Surg 2017;69:75-82.

15. Eriksson LSE, Lindqvist PG, Flöter Rådestad A, et al. Transvaginal ultrasound assessment of myometrial and cervical stromal invasion in women with endometrial cancer: interobserver reproducibility among ultrasound experts and gynecologists. Ultrasound Obstet Gynecol 2015;45:476-82.

16. Kakhki VR, Shahriari S, Treglia G, et al. Diagnostic performance of fluorine 18 fluorodeoxyglucose positron emission tomography imaging for detection of primary lesion and staging of endometrial cancer patients: systematic review and meta-analysis of the literature. Int J Gynecol Cancer 2013;23:1536-43.

17. Franchi M, Garzon S, Zorzato PC, et al. PET-CT scan in the preoperative workup of early stage intermediate- and high-risk endometrial cancer. Minim Invasive Ther Allied Technol 2020;29:232-9.

18. Casarin J, Bogani G, Serati M, et al. Presence of Glandular Cells at the Preoperative Cervical Cytology and Local Recurrence in Endometrial Cancer. Int J Gynecol Pathol 2020;39:522-8.

19. AlHilli MM, Mariani A. The role of para-aortic lymphadenectomy in endometrial cancer. Int J Clin Oncol 2013;18:193-9.

20. Creasman WT, Morrow CP, Bundy BN, et al. Surgical pathologic spread patterns of endometrial cancer. A Gynecologic Oncology Group Study. Cancer 1987;60:2035-41.

21. Milam MR, Java J, Walker JL, et al. Nodal metastasis risk in endometrioid endometrial cancer. Obstet Gynecol 2012;119:286-92.

22. Mariani A, Webb MJ, Keeney GL, et al. Low-risk corpus cancer: is lymphadenectomy or radiotherapy necessary? Am J Obstet Gynecol 2000;182:1506-19.

23. Mariani A, Dowdy SC, Cliby WA, et al. Prospective assessment of lymphatic dissemination in endometrial cancer: a paradigm shift in surgical staging. Gynecol Oncol 2008;109:11-8.

24. Todo Y, Kato H, Kaneuchi M, et al. Survival effect of paraaortic lymphadenectomy in endometrial cancer (SEPAL study): a retrospective cohort analysis. Lancet (London, England) 2010;375:1165-72.

25. Kitchener H, Swart AMC, Qian Q, et al. Efficacy of systematic pelvic lymphadenectomy in endometrial cancer (MRC ASTEC trial): a randomised study. Lancet (London, England) 2009;373:125-36.

26. Benedetti Panici P, Basile S, Maneschi F, et al. Systematic pelvic lymphadenectomy vs. no lymphadenectomy in early-stage endometrial carcinoma: randomized clinical trial. J Natl Cancer Inst 2008;100:1707-16.

27. Frost JA, Webster KE, Bryant A, et al. Lymphadenectomy for the management of endometrial cancer. Cochrane database Syst Rev 2017;10:CD007585.

28. Casarin J, Multinu F, Abu-Rustum N, et al. Factors influencing the adoption of the sentinel lymph node technique for endometrial cancer staging: an international survey of gynecologic oncologists. Int J Gynecol Cancer 2019;29:60-7.

29. Ballester M, Dubernard G, Lécuru F, et al. Detection rate and diagnostic accuracy of sentinel-node biopsy in early stage endometrial cancer: a prospective multicentre study (SENTI-ENDO). Lancet Oncol 2011;12:469-76. 
30. Barlin JN, Khoury-Collado F, Kim CH, et al. The importance of applying a sentinel lymph node mapping algorithm in endometrial cancer staging: beyond removal of blue nodes. Gynecol Oncol 2012;125:531-5.

31. Pelosi E, Arena V, Baudino B, et al. Preliminary study of sentinel node identification with $99 \mathrm{~m}$ Tc colloid and blue dye in patients with endometrial cancer. Tumori 2002;88:S9-10.

32. Rossi EC, Ivanova A, Boggess JF. Robotically assisted fluorescence-guided lymph node mapping with ICG for gynecologic malignancies: a feasibility study. Gynecol Oncol 2012;124:78-82.

33. Frumovitz M, Plante M, Lee PS, et al. Near-infrared fluorescence for detection of sentinel lymph nodes in women with cervical and uterine cancers (FILM): a randomised, phase 3, multicentre, non-inferiority trial. Lancet Oncol 2018;19:1394-403.

34. Abu-Rustum NR. Sentinel lymph node mapping for endometrial cancer: a modern approach to surgical staging. J Natl Compr Canc Netw 2014;12:288-97.

35. Martinelli F, Ditto A, Bogani G, et al. Sentinel lymph node mapping in endometrial cancer: performance of hysteroscopic injection of tracers. Int J Gynecol Cancer 2020;30:332-8.

36. Rossi EC, Kowalski LD, Scalici J, et al. A comparison of sentinel lymph node biopsy to lymphadenectomy for endometrial cancer staging (FIRES trial): a multicentre, prospective, cohort study. Lancet Oncol 2017;18:384-92.

37. Soliman PT, Westin SN, Dioun S, et al. A prospective validation study of sentinel lymph node mapping for highrisk endometrial cancer. Gynecol Oncol 2017;146:234-9.

38. Bogani G, Mariani A, Paolini B, et al. Low-volume disease in endometrial cancer: The role of micrometastasis and isolated tumor cells. Gynecol Oncol 2019;153:670-5.

39. Zahl Eriksson AG, Ducie J, Ali N, et al. Comparison of a sentinel lymph node and a selective lymphadenectomy algorithm in patients with endometrioid endometrial carcinoma and limited myometrial invasion. Gynecol Oncol 2016;140:394-9.

Cite this article as: Pinelli C, Artuso V, Bogani G, Laganà AS, Ghezzi F, Casarin J. Lymph node evaluation in endometrial cancer: how did it change over the last two decades? Transl Cancer Res 2020;9(12):7778-7784. doi: 10.21037/tcr-20-2165
40. Multinu F, Casarin J, Cappuccio S, et al. Ultrastaging of negative pelvic lymph nodes to decrease the true prevalence of isolated paraaortic dissemination in endometrial cancer. Gynecol Oncol 2019;154:60-4.

41. Gómez-Hidalgo NR, Ramirez PT, Ngo B, et al. Oncologic impact of micrometastases or isolated tumor cells in sentinel lymph nodes of patients with endometrial cancer: a meta-analysis. Clin Transl Oncol 2020;22:1272-9.

42. Plante M, Stanleigh J, Renaud MC, et al. Isolated tumor cells identified by sentinel lymph node mapping in endometrial cancer: Does adjuvant treatment matter? Gynecol Oncol 2017;146:240-6.

43. Kumar S, Medeiros F, Dowdy SC, et al. A prospective assessment of the reliability of frozen section to direct intraoperative decision making in endometrial cancer. Gynecol Oncol 2012;127:525-31.

44. Casarin J, Multinu F, Pasupathy K, et al. Frozen Section for Detection of Lymph Nodes After Cervical Injection with Indocyanine Green (ICG) for Sentinel Lymph Node Technique in Endometrial Cancer Staging. Ann Surg Oncol 2018;25:3692-8.

45. Bogani G, Murgia F, Ditto A, et al. Sentinel node mapping vs. lymphadenectomy in endometrial cancer: A systematic review and meta-analysis. Gynecol Oncol 2019;153:676-83.

46. Bogani G, Casarin J, Maggiore ULR, et al. Survival outcomes in endometrial cancer patients having lymphadenectomy, sentinel node mapping followed by lymphadectomy and sentinel node mapping alone: Longterm results of a propensity-matched analysis. Gynecol Oncol 2020;158:77-83.

47. Kandoth C, Schultz N, Cherniack AD, et al. Integrated genomic characterization of endometrial carcinoma. Nature 2013;497:67-73.

48. Wortman BG, Bosse T, Nout RA, et al. Molecularintegrated risk profile to determine adjuvant radiotherapy in endometrial cancer: Evaluation of the pilot phase of the PORTEC-4a trial. Gynecol Oncol 2018;151:69-75. 